\title{
Risk Factors for Adverse Reactions During OIT
}

Nandinee Patel ${ }^{1}$

Marta Vazquez-Ortiz ${ }^{1}$

Paul J. Turner ${ }^{1,2, *}$

\author{
Address \\ *,1 Section of Paediatrics (Allergy \& Infectious Diseases), Imperial College London, \\ Norfolk Place, London, W2 1PG, UK \\ Email: p.turner@imperial.ac.uk \\ ${ }^{2}$ Discipline of Paediatrics and Child Health, School of Medicine, University of \\ Sydney, Sydney, 2000, Australia
}

Published online: 1 May 2019

(C) The Author(s) 2019

\section{Electronic supplementary material}

This article is part of the Topical Collection on Food Allergy

The online version of this article (https://doi.org/10.1007/s40521-019-00205-2) contains supplementary material, which is available to authorized users.

Keywords Oral immunotherapy $\cdot$ Risk factors $\cdot$ Safety $\cdot$ Predicting adverse reactions

\begin{abstract}
Purpose of review Oral immunotherapy (OIT) can have a major positive impact on patients with IgE-mediated food allergies, increasing reaction thresholds and reducing the need for dietary and lifestyle limitations. However, patients experience more frequent allergic reactions during 0IT than when following dietary avoidance, and $10-75 \%$ of patients on OIT may experience anaphylaxis to treatment doses. Our ability to identify patients at higher risk of more severe or frequent reactions during OIT is limited. We review the current data available and highlight the gaps in knowledge which impede our ability to predict response to treatment, occurrence of dose-related adverse events, and thus acceptance of 0IT into wider clinical practice.

Recent findings Our ability to predict the risk of severe reactions in food-allergic patients is limited, due to the multitude of allergen and host-related factors which influence this. While OIT is thought to reduce this risk, little is known about the immunomodulatory effect of OIT on these factors, and the resulting risk of allergic events during 0IT. Several factors have been associated with reaction severity during 0IT and treatment withdrawals, including high allergen-specific IgE levels and certain IgE epitope binding patterns. Other factors proposed include the degree of sensitisation on skin testing, initial reaction threshold, prior reaction severity, age, and concomitant allergic disease including allergic rhinitis and asthma. These have also been associated with more severe events in foodallergic patients not undergoing $0 \mathrm{IT}$, and suggest a specific patient phenotype prone to more severe and persistent food allergy, which also impact on poorer outcomes during 0IT.
\end{abstract}


Ironically, it is this patient phenotype that arguably has most to gain from 0IT. Summary Our understanding of the constellation of factors contributing to reaction threshold, nature, and severity in food allergy is improving, and this helps understand the complexity of OIT safety. Potential predictors of OIT safety are becoming available. However, accurate prediction cannot be done at individual level at present, and significant concerns around OIT safety remain, arguably making this treatment unsuitable for routine practice. Predictors are likely to be study- and population-specific, given the variation in OIT protocols, patient characteristics, and adverse event reporting. Consensus is needed on detailed adverse event reporting, and pooled analysis of multiple series is likely to yield more useful predictors of OIT safety.

\section{Introduction}

Oral immunotherapy (OIT) was first trialled as a potential disease-modifying treatment for IgE-mediated food allergy in 1908 [1], but until recently, there had been a relative hiatus in establishing OIT as a viable treatment modality. There is proven efficacy from numerous phase $2 / 3$ trials where outcome measures have largely centred on the change in reaction threshold at supervised food challenges using incremental doses and (in some studies) improvements in health-related quality of life measures, although, typically, these are parent-reported. Although the key mechanisms of OIT are poorly understood, OIT has become increasingly offered within clinical practice in several countries [2, 3], though details remain limited in many countries. [4]. More recently, larger phase 3 studies are also now underway, as are studies looking at potential alternative routes of administration [5-7]. There is increasing interest from the commercial sector to develop immunotherapy products for food allergy, given public demand.

Allergen immunotherapy carries an inherent risk of allergic reactions, as dosing regimens need to balance a level of allergen exposure sufficient to induce immune desensitisation but low enough not to trigger an IgEmediated reaction. The most important knowledge gap-and arguably the main barrier to regulatory approval-remains the safety profile (both short and longer term) of OIT in the treatment of IgE-mediated food allergies [8]. Studies report a rate of allergic reaction affecting between 18 and $100 \%$ of patients on active OIT, with $0.8-43 \%$ of doses causing symptoms [9-11]. This may lead to OIT failure, with $5-36 \%$ of patients being withdrawn during treatment $[11,12]$. Immunotherapy has the potential to cause fatality through a severe adverse reaction, either during the initial screening challenges prior to treatment or dosing-related reactions [13].

With such wide variations in safety outcomes, being able to predict those at risk of greater and more severe adverse events is important. These factors can be divided into:

1) Those that are patient-dependent, where selective characteristics place a specific individual at higher or lower risk of AEs.

2) Features that are related to the protocol, that may affect the likelihood of dose-related AEs across study participants.

OIT efficacy has been evaluated in a number of systematic reviews, but analyses of dose-related adverse events (AEs) and other safety outcomes-both in relation to patient-dependent factors and those inherent to the protocol-have proved more problematic due to lack of consistency in reporting adverse events [14]. A meta-analysis by Nurmatov included 21 clinical trials of which 17 used OIT (with a predominance of studies assessing cow's milk OIT), and found (perhaps unsurprisingly) that active OIT results in a greater risk of local and systemic allergic reactions compared to control treatments [15]. The authors attempted to evaluate the impact of different protocols and food allergens, but this was limited by the heterogeneity of reporting in the different studies. In consequence, only seven trials could be 
included in the analysis; the authors were unable to identify any factors associated with adverse events during immunotherapy, despite anecdotal reports suggesting milk and wheat immunotherapy often result in more frequent and more severe adverse events [16, 17].

In this article, we review the published data relating to the occurrence of dose-related adverse events and the inherent issues with such an analysis. Importantly, this needs to be framed in the wider context of our inability to predict reaction severity in food-allergic individuals [18] in a clinically meaningful way, and how this may be further confounded by the immune changes induced by OIT, resulting in unexpected breakthrough reactions [19].

\section{Difficulties in interpreting safety data}

Evaluating the differences in datasets generated by OIT studies in order to assess which factors might impact on safety is hampered by:

1. Heterogeneity in patients recruited: Differences in the eligibility criteria for study participants can be a major confounder in the analysis of safety data from studies. Some studies use a relatively high cutoff for allergen-specific IgE sensitisation as an inclusion criteria (e.g., 7 kUA/l [20]) compared with others which allow any level of sensitisation, something which might impact on the occurrence of AEs as there is some data to show that these are more common with higher levels of sensitisation. Historically, exclusion criteria have sometimes excluded those with a history of anaphylaxis [21], anaphylaxis (with respiratory symptoms) at baseline challenge [22]; most studies (probably quite reasonably) continue to exclude those with prior severe reaction requiring intensive care.

2. Heterogeneity in protocol design:

(a) OIT protocols tend to consist of an initial updosing phase followed by a maintenance phase. More rapid updosing (e.g., "rush" updosing) or protocols where duration of updosing is shorter may be associated with a higher rate of AEs [8], although no head-to-head comparison has as yet been published.

(b) Protocols vary in terms of the allergen "matrix" used to induce desensitisation: for example, peanut OIT protocols typically use defatted roasted peanut flour, but some studies have utilised roasted (not defatted) peanut in either ground or solid form, while others have used heatmodified peanut [23]. The different preparations may impact upon the occurrence of AEs, but thus confound any comparison between studies.

(c) Many studies do not include a control group, and those that do may utilise routine care (i.e., allergen avoidance) rather than a true placebo control; studies incorporating a blinded placebo intervention tend to report higher rates of AEs than mere avoidance. While efficacy of active treatments should be compared with a placebo intervention, whether this holds true with respect to safety outcomes is less clear. For example, if a study reports a $50 \%$ rate of gastrointestinal (GI) events with active treatment, and $15 \%$ with a placebo intervention is the true rate of GI events nearer to $35 \%$ rather than $50 \%$ ?

(d) Studies also vary in the safety precautions taken, which impacts upon outcomes including occurrence of AEs. Some protocols include provision to temporarily cease dosing for fever or gastro-intestinal symptoms [22], 
while others mandate dose-reductions following dose-related symptoms. Thus, the occurrence of AEs will vary depending on the strategies used to manage dose-related symptoms during OIT, and may be clinician-

dependent where the protocols provide flexibility to study investigators in terms of the available options.

3. Heterogeneity in AE reporting: Unfortunately, there is no current consensus on AE reporting across studies. Many studies only provide data relating to serious AEs requiring treatment with adrenaline [24], although adrenaline use is a poor marker of reaction severity [25].

In contrast to studies of aeroallergen immunotherapy [26], there is no current consensus in terms of reporting structures for studies of OIT. Differences in data collection between studies (e.g., prospective vs retrospective, paper vs electronic reporting) and assignment of impact/severity are then further confounded by variations in reporting; of the 23 studies reviewed for this article (Table E1), only 12 (52\%) reported the total number of doses administered, 15 (65\%) the number of patients experiencing at least one AE during OIT, 10 (43\%) the proportion of doses causing AEs, and 13 (57\%) a severity grading for reactions. Reporting AEs using number of doses as the denominator gives the perception that $\mathrm{AE}$ are less common; in the STOP-2 study [27], there were 73 episodes of wheezing-reported as an AE in relation to a dose of OIT-in 21 patients. Is this best reported as $0.41 \%$ of doses administered, or as an $\mathrm{AE}$ which occurred in $22 \%$ of participants? Or should both figures be presented (as the study investigators chose to do), in order to address this issue?

\section{Improving the safety of OIT}

The need for consistency in AE collections and reporting is essential if we are going to be able to attempt even limited comparisons between different study protocols, with the aim to improve patient safety and efficacy outcomes. We have previously reviewed the options to improve the safety of OIT [8], and these can be summarised as strategies to:

a) improve protocol designs - by use of hypoallergenic products (e.g., through food-processing or selection of specific peptides), modification of schedule intensity or the use of adjuvants such as omalizumab.

b) improve patient selection, in order to offer individualised protocols which are better targeted to patients with "high-risk" profiles who are considered to be at greater risk of dose-related AEs and treatment failure.

This requires a two-pronged approach: one that aims to increase overall safety of OIT, and second, to specifically identify patients who might be at higher risk of AEs and treatment failure. Given finite resources, the focus should be on the predictability and prevention of (1) high-risk adverse events (anaphylaxis, recurrent gastrointestinal AEs) and (2) reactions during longer-term maintenance, where it is not uncommon for patients who have tolerated a daily dose for many weeks and even months to suddenly experience a breakthrough reaction including anaphylaxis.

\section{Factors potentially contributing to dose-related AEs}

These may be divided into three broad categories, as summarised in Fig. 1. 


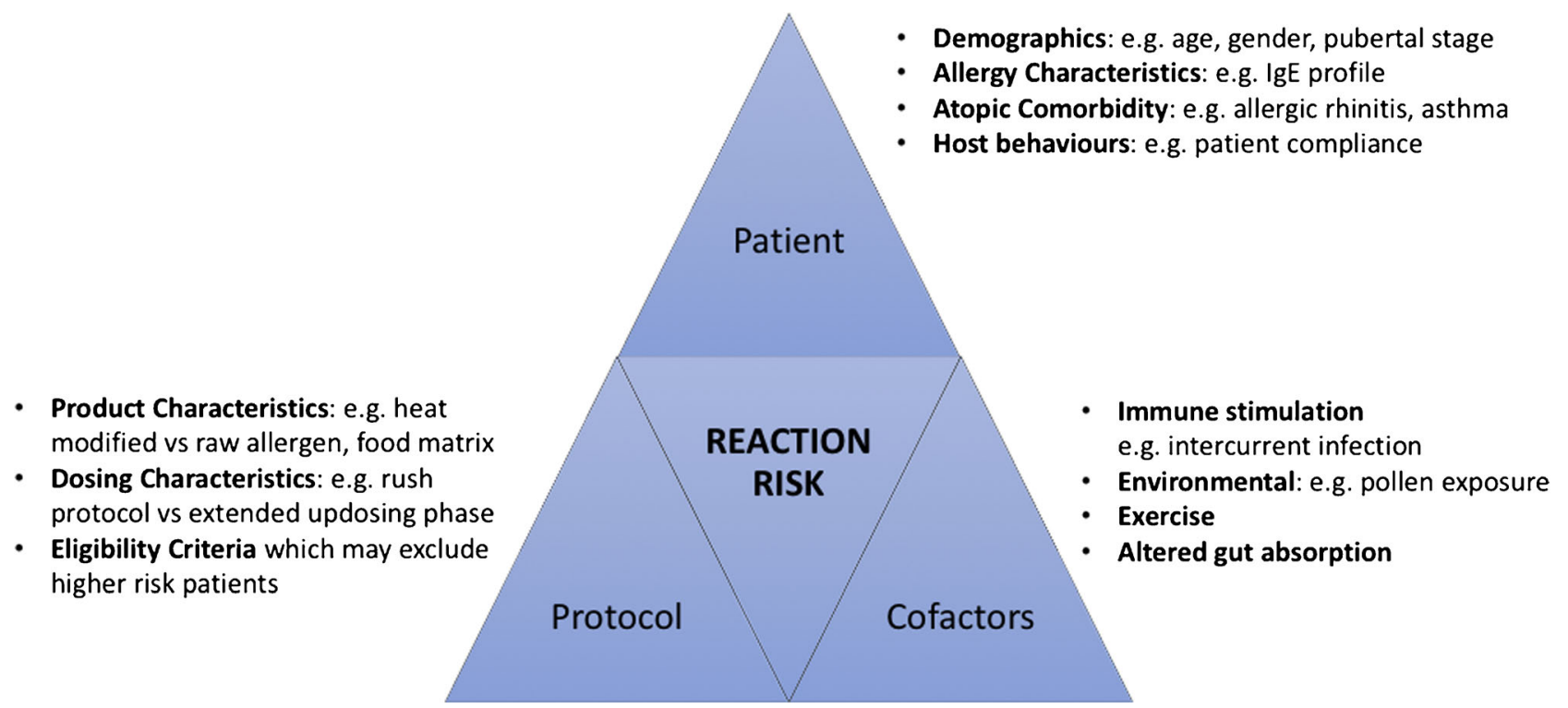

Fig. 1. Potential factors which may contribute to the occurrence and severity of dose-related adverse events during 0IT

1. Protocol-related factors

Several early studies used initial rush updosing phases which were associated with high $\mathrm{AE}$ rates [13]. However, this might have led to earlier treatment failures, with the consequence of selecting out higher-risk patients, thus reducing the overall dose-related $\mathrm{AE}$ rates during subsequent treatment phases. Many protocols now include a "rush" updosing phase over several days [28-30], although evidence that this is a more efficacious approach than slower, more extended initiation phases is lacking, as no head-to-head comparison has been undertaken.

Dello Iacono et al. reported that frequency (but not severity) of AEs correlated with the size of increment at any given dose-step in an open-label trial of egg OIT [31]. The study team proposed that the higher rate of in-hospital AEs was a function of higher dosing increments in hospital compared with those undertaken at home. However, it is difficult to avoid confounding due to better reporting of in-hospital AEs compared with those at home, where minor symptoms might not always be recorded. In contrast, Vazquez-Ortiz et al. noted that for egg OIT, the greatest number of AEs occurred at the lowest and highest doses and did not necessarily relate to the dosing increment [32]. For peanut, Wasserman et al. did not observe any impact of dosing increment on risk of AEs [2].

Multiple studies have reported higher rates of AEs during the initial updosing phase, which reduce over time $[11,20]$. However, safety data relating to longer-term maintenance (where adherence may be reduced) are sparse. In the pooled analysis by Virkud et al. for peanut OIT, while overall rates for AEs were greater during the build-up phase, more patients received adrenaline (epinephrine) during maintenance phase (although the rate was not adjusted for number of doses in each phase and patient withdrawal); this might reflect increasing compliance with appropriate management of breakthrough reactions and/or reduced adherence to therapy [20].

Finally, rates of AEs may also be related to the target maintenance dose. Paradoxically, in a comparison of high and low dose peanut OIT, Vickery et al. 
reported a significantly greater rate of moderate AEs in the lower dose group, which reversed during maintenance [11]. This suggests that a higher target might protect against AEs by inducing a faster and larger increase in the dose needed to trigger AEs, thus providing greater protection from dosing-related AEs. Although, it has been proposed that lower-dose protocols might be a useful strategy in desensitising higher-risk patients [33], it is difficult to make conclusions due to the lack of consistency in reported AE data between studies. No statistical difference was observed in rates of AEs in a recent report of lower and higher-dose OIT for wheat allergy [34].

2. Patient-related factors

(a) Egg

Vazquez-Ortiz et al. reported that $90 \%$ of children $(n=50)$ receiving OIT to pasteurised raw egg experienced AEs [32]. Participants were divided into those with AEs that resolved over time (median time to resolution 7.6 months) $(48 \%)$, those with persistent reactions (34\%) and those who discontinued OIT due to side effects (18\%). Participants with persistent reactions had more frequent and more severe reactions, while those who discontinued treatment had higher specific baseline IgE and a lower threshold at baseline challenge.

For both egg and milk OIT, Garcia-Lirio et al. noted the serum cytokine levels (IL-2, IL-4, IL-6, IL-10, IL-12, IL-17, IFN $\gamma$, and TNF $\alpha$ ) did not change during OIT and therefore could not predict those patients who might be at greater risk of persistent reactions [35]. Unfortunately, the authors did not assess cytokine response of peripheral blood mononuclear cells following in vitro allergen stimulation.

(b) Cow's milk

In an open-label trial of milk-OIT, Salvilahti et al. reported a significant difference between baseline allergen-specific IgE and IgG4 levels, diversity and binding affinity (including the major component $\alpha$-1-casein) in those successfully completing OIT compared with those withdrawing due to AEs [36]. Interestingly, participants who failed OIT had higher affinity for both IgE and IgG4 at baseline, while those in whom OIT was successful had greater overlap in IgE and IgG4 regions prior to OIT initiation, compared with treatment failures. Importantly, the diversity of binding did not significantly alter over time, and thus might provide a baseline indicator to help predict outcome. In another study, Martinez-Botas et al. found a linear association between the number of peptides demonstrating IgE binding and the number of AEs during milk-OIT [16].

Vazquez-Ortiz et al. undertook survival analysis in a cohort of 81 Spanish children undergoing cow's milk OIT; the cumulative probability of resolution of dose-related AEs was $25 \%$ and $50 \%$ at 3 and 8 months respectively [37]. Allergen-specific IgE $>50 \mathrm{kua} / \mathrm{L}$, skin prick wheal $>9 \mathrm{~mm}$, and more severe reactions at pre-OIT food challenge were found to be independent risk factors for persisting AEs during OIT, with an additive/synergistic risk elevation on combination of risk factors.

Finally, Kuitunen et al. investigated predictors of treatment efficacy in a study of 76 children undergoing OIT to cow's milk [38]. They found that participants who were classified as partial responders (tolerating $<200 \mathrm{ml}$ cow's milk at exit challenge) had a significantly higher proportion of adverse skin reactions during treatment, compared with those who tolerated $>200 \mathrm{ml}$ following OIT ( $43 \%$ vs $18 \%$ of all adverse reactions, $p=0.038$ ). 


\section{(c) Peanut}

Virkud et al. [20] undertook a pooled analysis of data from three studies of peanut OIT (two of which were not included in the meta-analysis by Nurmatov et al. [15]), with a total of 104 children included. Rates of systemic AEs increased 2.2 times (95\% CI 1.1-4.3) in participants with allergic rhinitis, compared with those without. Patients with allergic rhinitis experienced doserelated AEs more frequently between April and October i.e., seasonality, while those without experienced more AEs between January and September in those without allergic rhinitis. However, whether this observation remained after correcting for date of OIT commencement and/or duration on OIT is unclear. A significant relation was noted between skin sensitisation and gastrointestinal AEs, with a 1.8-fold increase in gastrointestinal events for every $5 \mathrm{~mm}$ increase in baseline SPT size. However, 92\% of this cohort were under 8 years old (55\% under 3 years), so these findings need replication, both in the same and older age groups. Likewise, some of the included studies had a peanut-specific IgE $\geq$ $7 \mathrm{kUA} / \mathrm{l}$ as an inclusion criteria; as a result, only $12 \%$ of participants had a peanut-specific IgE $<7 \mathrm{kUA} / \mathrm{L}$, and so the adverse event frequency might be higher than in the general peanut-allergic population.

Wasserman et al's recent analysis of 270 patients undergoing office-based OIT reported that the odds of achieving successful desensitisation (defined as tolerance to a maintenance dose of $3 \mathrm{~g}$ peanut protein and "passing" a food challenge of $6 \mathrm{~g}$ peanut protein) were reduced with increasing age and higher baseline peanut-specific IgE [2]. These data are consistent with the conclusions of another study by Vickery et al. who undertook peanut OIT study in a younger cohort (aged 9 to 36 months) [11], though direct comparison is difficult due to differences in protocols and definitions of adverse reactions. As of the time of writing, no analysis of predictors of dose-related AEs has yet been published for the PALISADE study, currently the largest phase 3 randomised controlled study of peanut OIT to date [39].

(d) Wheat

Kulmala recently published results from an open-label OIT study in 100 wheat-allergic children [17]. The authors report that baseline sensitisation to $\omega$ 5-gliadin but not whole wheat was associated with more severe AEs, although they note that some patients had very high baseline levels of antibodies $\omega$-5gliadin and yet had mild or even no reactions during the study. A smaller but randomised double-blind placebo-controlled study did not assess factorsassociated AEs, but did report a significant rate of AEs in the placebo group (5.8\% of all doses) compared with active treatment (15.4\%) which reinforces the need to assess for placebo reactions when interpreting AE data [34].

3. Co-factors

It is not uncommon for participants undergoing OIT to experience breakthrough reactions to a maintenance dose, despite having tolerated that dose for weeks or even months prior. Potential cofactors contributing to this can include intercurrent infections, exercise, tiredness, and menstruation [19], and it has been reported that most AEs during maintenance occurred in the context of cofactors [40]. However, it is difficult to determine the degree (if any) to which these factors contribute to the occurrence of the reaction, or whether their presence is just coincidental. There is a lack of data in relation to how frequently doses are tolerated despite the presence of these co-factors, which impedes these types of analysis. This 
remains a significant data gap, as it would be helpful to address, where there is a subset of patients who experience cofactor-related AEs and whom can be predicted in advance, thus reducing the need for dosing-related restrictions for other patients.

\section{Can we identify higher-risk patients as a strategy to improve the safety of 0IT?}

Unfortunately, around $20 \%$ of patients fail OIT; these patients tend to experience a higher frequency of dose-related AEs but also share the characteristics associated with a tendency towards more severe reactions with accidental allergen exposure [18]. Given these are the patients most likely to benefit from OIT, there is a need to find strategies to improve the treatment safety profile through modified protocols $[8,41]$. However, there are also some patients in whom the risk of current OIT protocols outweighs the risk associated with avoidance [42]. OIT is associated with more reactions than avoidance [43], although arguably for most patients undergoing OIT the increase in reaction frequency is still associated with an improvement in health-related quality of life.

Severe and life-threatening reactions during OIT have been reported [41, 42]. These patients are often adolescents (in whom compliance may be an issue) and have higher IgE levels and persistent asthma along with a history of recent missed doses or co-factors. However, these features cannot distinguish from many more children with the same characteristics, who safely achieve desensitisation. These and other patients with persistent food allergies may have specific epitope binding patterns, which could also signal a patient-group at higher risk of dose-related AEs and reduced efficacy during OIT, but this has

Table 1. Suggested minimum reported of AEs in studies of OIT

\begin{tabular}{|c|c|c|}
\hline & \multicolumn{2}{|c|}{ Overall and breakdown by phase } \\
\hline & $\begin{array}{l}\text { Reported proportion of } \\
\text { patients }\end{array}$ & $\begin{array}{l}\text { Reported proportion of doses } \\
\text { administered }\end{array}$ \\
\hline Withdrawals due to AEs & $x$ & \\
\hline Withdrawals to concerns over adherence & $x$ & \\
\hline Hospitalisation and other SAEs & $x$ & $x$ \\
\hline \multicolumn{3}{|l|}{ AEs by: } \\
\hline Severity & $x$ & $x$ \\
\hline $\begin{array}{l}\text { Treatment administered e.g. } \\
\text { antihistamine, IM adrenaline, steroids }\end{array}$ & $x$ & $x$ \\
\hline Symptoms/organ system involved & $x$ & $x$ \\
\hline AEs with suspected co-factor involvement & $x$ & $x$ \\
\hline Number and timing of delayed reactions & $x$ & $x$ \\
\hline AEs by location: in hospital vs home & $x$ & $x$ \\
\hline AEs by dose involved & $x$ & $x$ \\
\hline Non-dose related $A E s$ & $x$ & $x$ \\
\hline $\begin{array}{l}\text { AEs related to other allergic condition (as a marker } \\
\text { of disease control) }\end{array}$ & $x$ & $x$ \\
\hline
\end{tabular}




\section{Conclusions}

only, as yet, been assessed for milk OIT [44].

Given the differences in protocols for published and ongoing OIT trials, one potential way forward would be to undertake sequential meta-analyses and grouped analyses. Meta-analyses have been published relating to OIT efficacy, but this has proven to be less feasible with respect to safety outcomes. A consensus would be needed to ensure alignment of key data outcomes and standardised reporting of OIT data. Such a consensus statement would require input from all stakeholders-most importantly, patients and their families-in order to consider the relevance of outcomes which bother patients most, rather than study investigators who may impose their own values assessment on the impact of AEs occurring in our patients. This would also provide an opportunity to better align AE terms with the medical dictionary for regulatory activities, which would be of use in moves towards introducing OIT more widely in clinical practice from the regulatory perspective [45]. A suggested minimum data set is proposed in Table 1.

The lack of consistency in dose-related AE reporting in studies of OIT has impeded our understanding of the safety of OIT, including the factors which might contribute to differences in safety outcomes and which strategies might help improve this, particularly for patients who are more likely to fail OIT or experience frequent AEs. Many of the OIT trials reported to date are limited by small cohort size, which makes generalisation of safety data to the wider foodallergic population difficult. Consensus is needed on AE reporting to allow pooled analyses which are likely to yield more useful predictors of OIT safety, and lead to improved protocols to maximise efficacy and safety to the benefit of our patients.

\section{Compliance with ethical standards}

\section{Conflict of interest}

Paul Turner is in receipt of a Clinician Scientist award funded by the UK Medical Research Council (reference MR/ K010468/1). All authors have received support from the National Institute for Health Research (NIHR) Biomedical Research Centre based at the Imperial College Healthcare NHS Trust and Imperial College London. The views expressed are those of the authors and not necessarily those of the NHS, NIHR, or the Department of Health.

\section{Human and animal rights and informed consent}

This article does not contain any studies with human or animal subjects performed by any of the authors.

Open Access This article is distributed under the terms of the Creative Commons Attribution 4.0 International License (http://creativecommons.org/licenses/by/4.0/), which permits unrestricted use, distribution, and reproduction in any medium, provided you give appropriate credit to the original author(s) and the source, provide a link to the Creative Commons license, and indicate if changes were made. 
Papers of particular interest, published recently, have been highlighted as:

- Of importance

- Of major importance

1. Schofield AT. A case of egg poisoning. Lancet. 1908; $171: 716$.

2. Wasserman RL, Factor JM, Baker JW, Mansfield LE, Katz $\mathrm{Y}$, Hague AR, et al. Oral immunotherapy for peanut allergy: multipractice experience with epinephrinetreated reactions. J Allergy Clin Immunol. Pract. 2014;2(1):91-6.

3. Mansfield LE. Oral immunotherapy for peanut allergy in clinical practice is ready. Allergy and asthma proceedings. 2013;34(3):205-9.

4. Sato S, Sugizaki C, Yanagida N, Ito K, Ohshima Y, Shimojo N, et al. Nationwide questionnaire-based survey of oral immunotherapy in Japan. Allergol Int. 2018;67(3):399-404.

5. Jones SM, Sicherer SH, Burks AW, Leung DY, Lindblad RW, Dawson P, et al. Epicutaneous immunotherapy for the treatment of peanut allergy in children and young adults. J Allergy Clin Immunol. 2017;139(4):1242-52e9.

6. Fleischer DM, Burks AW, Vickery BP, Scurlock AM, Wood RA, Jones SM, et al. Sublingual immunotherapy for peanut allergy: a randomized, double-blind, placebo-controlled multicenter trial. J Allergy Clin Immunol. 2013;131(1):119-27.e1-7.

7. Keet CA, Frischmeyer-Guerrerio PA, Thyagarajan A, Schroeder JT, Hamilton RG, Boden S, et al. The safety and efficacy of sublingual and oral immunotherapy for milk allergy. J Allergy Clin Immunol.

2012;129(2):448-5555 e1-5.

8. Vazquez-Ortiz M, Turner PJ. Improving the safety of oral immunotherapy for food allergy. Pediatric Allergy Immunol. 2016;27(2):117-25.

9. Caminiti L, Pajno GB, Crisafulli G, Chiera F, Collura M, Panasci G, et al. Oral immunotherapy for egg allergy: a double-blind placebo-controlled study, with postdesensitization follow-up. J Allergy Clin Immunol. Pract. 2015;3(4):532-9.

10. Narisety SD, Frischmeyer-Guerrerio PA, Keet CA, Gorelik M, Schroeder J, Hamilton RG, et al. A randomized, double-blind, placebo-controlled pilot study of sublingual versus oral immunotherapy for the treatment of peanut allergy. J Allergy Clin Immunol. 2015;135(5):1275-82 e1-6.

11. Vickery BP, Berglund JP, Burk CM, Fine JP, Kim EH, Kim JI, et al. Early oral immunotherapy in peanutallergic preschool children is safe and highly effective. J Allergy Clin Immunol. 2017;139(1):173-81 e8.

12. Andorf S, Manohar M, Dominguez T, BlockW, Tupa D, Kshirsagar RA, et al. Observational long-term followup study of rapid food oral immunotherapy with omalizumab. Allergy Asthma Clin Immunol. 2017;13:51.

13. Oppenheimer JJ, Nelson HS, Bock SA, Christensen F, Leung DY. Treatment of peanut allergy with rush immunotherapy. J Allergy Clin Immunol. 1992;90(2):256-62.

14. Nurmatov U, Venderbosch I, Devereux G, Simons FE, Sheikh A. Allergen-specific oral immunotherapy for peanut allergy. Cochrane Database Syst Rev. 2012 12;(9):CD009014. https://doi.org/10.1002/ 14651858.CD009014.pub2.

15.• Nurmatov U, Dhami S, Arasi S, Pajno GB, FernandezRivas M, Muraro A, et al. Allergen immunotherapy for IgE-mediated food allergy: a systematic review and meta-analysis. Allergy. 2017;72(8):1133-47.

Comprehensive meta-analysis and systematic review of immunotherapy studies for food allergy

16. Martinez-Botas J, Rodriguez-Alvarez M, Cerecedo I, Vlaicu C, Dieguez MC, Gomez-Coronado D, et al. Identification of novel peptide biomarkers to predict safety and efficacy of cow's milk oral immunotherapy by peptide microarray. Clin Exp Allergy. 2015;45(6):1071-84.

17. Kulmala P, Pelkonen AS, Kuitunen M, Paassilta M, Remes S, Schultz R, et al. Wheat oral immunotherapy was moderately successful but was associated with very frequent adverse events in children aged 6-18 years. Acta Paediatr. 2018;107(5):861-70.

18. Turner PJ, Baumert JL, Beyer K, et al. Can we identify patients at risk of life-threatening allergic reactions to food? Allergy. 2016;71(9):1241-55.

A European consensus on our current (in)ability to predict severity in food allergy.

19. Anagnostou K, Clark A, King Y, Islam S, Deighton J, Ewan P. Efficacy and safety of high-dose peanut oral immunotherapy with factors predicting outcome. Clin Exp Allergy. 2011;41(9):1273-81.

20. Virkud YV, Burks AW, Steele PH, Edwards LJ, Berglund JP, Jones SM, et al. Novel baseline predictors of adverse events during oral immunotherapy in children with peanut allergy. J Allergy Clin Immunol. 2017;139(3):882-8 e5.

21. Buchanan AD, Green TD, Jones SM, Scurlock AM, Christie L, Althage KA, et al. Egg oral immunotherapy in nonanaphylactic children with egg allergy. J Allergy Clin Immunol. 2007;119(1):199-205.

22. Akashi M, Yasudo H, Narita M, Nomura I, Akasawa A, Ebisawa $\mathrm{M}$, et al. Randomized controlled trial of oral immunotherapy for egg allergy in Japanese patients. Pediatr Int. 2017;59(5):534-9.

23. Turner PJ, Mehr S, Sayers R, et al. Loss of allergenic proteins during boiling explains tolerance to boiled peanut in peanut allergy. J Allergy Clin Immunol. 2014;134:751-3. 
24. Varshney P, Jones SM, Scurlock AM, Perry TT, Kemper A, Steele P, et al. A randomized controlled study of peanut oral immunotherapy: clinical desensitization and modulation of the allergic response. J Allergy Clin Immunol. 2011 Mar;127(3):654-60.

25. Santos AF, Du Toit G, Lack G. Is the use of epinephrine a good marker of severity of allergic reactions during oral food challenges? J Allergy Clin Immunol Pract. 2015;3(3):429-30.

26. Pfaar O, Demoly P, Gerth van Wijk R, Bonini S, Bousquet J, Canonica GW, et al. European Academy of Allergy and Clinical Immunology. Recommendations for the standardization of clinical outcomes used in allergen immunotherapy trials for allergic rhinoconjunctivitis: an EAACI Position Paper. Allergy. 2014;69(7):854-67.

27. Anagnostou K, Islam S, King Y, et al. Assessing the efficacy of oral immunotherapy for the desensitisation of peanut allergy in children (STOP II): a phase 2 randomised controlled trial. Lancet. 2014;383:1297304.

28. Garcia Rodriguez R, Urra JM, Feo-Brito F, Galindo PA, Borja J, Gomez E, et al. Oral rush desensitization to egg: efficacy and safety. Clin Exp Allergy. 2011;41(9):128996.

29. Itoh N, Itagaki Y, Kurihara K. Rush specific oral tolerance induction in school-age children with severe egg allergy: one year follow up. Allergol Int.

2010;59(1):43-51.

30. Perez-Rangel I, Rodriguez Del Rio P, Escudero C, Sanchez-Garcia S, Sanchez-Hernandez JJ, Ibanez MD. Efficacy and safety of high-dose rush oral immunotherapy in persistent egg allergic children: a randomized clinical trial. Annals Allergy Asthma Immunol. 2017;118(3):356-64.e3.

31. Dello Iacono I, Tripodi S, Calvani M, Panetta V, Verga MC, Miceli Sopo S. Specific oral tolerance induction with raw hen's egg in children with very severe egg allergy: a randomized controlled trial. Pediatr Allergy Immunol. 2013 Feb;24(1):66-74.

32. Vazquez-Ortiz M, Alvaro M, Piquer M, Dominguez $\mathrm{O}$, Machinena A, Martin-Mateos MA, et al. Baseline specific IgE levels are useful to predict safety of oral immunotherapy in egg-allergic children. Clin Exp Allergy. 2014;44(1):130-41.

33. Yanagida N, Sato S, Asaumi T, Okada Y, Ogura K, Ebisawa M. A single-center, case-control study of lowdose-induction oral immunotherapy with cow's milk. Int Arch Allergy Immunol. 2015;168(2):131-7.

34. Nowak-Wegrzyn A, Wood RA, Nadeau KC, Pongracic JA, Henning AK, Lindblad RW, et al. Multicenter, randomized, double-blind, placebo-controlled clinical trial of vital wheat gluten oral immunotherapy. J Allergy Clin Immunol. 2018. https://doi.org/10.1016/j. jaci.2018.08.041.

35. Garcia-Lirio E, Gonzalez Diaz C, Gonzalez Hermosa A, Gamboa P, Aranguren R, Sanz ML. Oral immunotherapy with egg and milk: changes in peripheral serum cytokines are not predictive factors for severe adverse reactions or for the final report. J Investig Allergol Clin Immunol. 2018;28(1):24-8.

36. Savilahti EM, Kuitunen M, Valori M, Rantanen V, Bardina L, Gimenez G, et al. Use of IgE and IgG4 epitope binding to predict the outcome of oral immunotherapy in cow's milk allergy. Pediatr Allergy Immunol. 2014;25(3):227-235.

37. Vazquez-Ortiz M, Alvaro-Lozano M, Alsina L, GarciaPaba MB, Piquer-Gibert M, Giner-Munoz MT, et al. Safety and predictors of adverse events during oral immunotherapy for milk allergy: severity of reaction at oral challenge, specific IgE and prick test. Clin Exp Allergy. 2013;43(1):92-102.

38. Kuitunen M, Englund H, Remes S, Moverare R, Pelkonen A, Borres MP, et al. High IgE levels to alphalactalbumin, beta-lactoglobulin and casein predict less successful cow's milk oral immunotherapy. Allergy. 2015;70(8):955-62.

39. PALISADE Group of Clinical Investigators, Vickery BP, Vereda A, Casale TB, et al. AR101 Oral Immunotherapy for Peanut Allergy. N Engl J Med. 2018;379(21):19912001.

40. Pajno GB, Caminiti L, Salzano G, Crisafulli G, Aversa T, Messina MF, et al. Comparison between two maintenance feeding regimens after successful cow's milk oral desensitization. Pediatric Allergy Immunol. 2013;24(4):376-81.

41. Vazquez-Ortiz M, Alvaro M, Piquer M, Giner MT, Dominguez O, Lozano J, et al. Life-threatening anaphylaxis to egg and milk oral immunotherapy in asthmatic teenagers. Annals Allergy Asthma Immunol. 2014;113(4):482-4.

42. Nieto A, Fernandez-Silveira L, Mazon A, Caballero L. Life-threatening asthma reaction caused by desensitization to milk. Allergy. 2010;65(10):1342-3.

43.• Shaker MS. An economic analysis of a peanut oral immunotherapy study in children. J Allergy Clin Immunol Pract. 2017;5:1707-16.

An analysis of the key considerations for attempting to model potential cost-benefit analyses of peanut immunotherapy in clinical practice.

44. Suárez-Fariñas M, Suprun M, Chang HL, Gimenez G, Grishina G, Getts R, et al. Predicting development of sustained unresponsiveness to milk oral immunotherapy using epitope-specific antibody binding profiles. J Allergy Clin Immunol. 2018. https://doi.org/10.1016/ j.jaci.2018.10.028.

45. Passalacqua G, Nowak-Węrzyn A, Canonica GW. Local side effects of sublingual and oral immunotherapy. J Allergy Clin Immunol Pract. 2017;5(1):13-21.

\section{Publisher's Note}

Springer Nature remains neutral with regard to jurisdictional claims in published maps and institutional affiliations. 